\title{
Estudo etnobotânico do conhecimento e uso de plantas medicinais em Santa Luzia, Maranhão, Brasil
}

O uso de plantas como medicamentos tradicionais fornece um substituto real nos serviços de saúde para as comunidades rurais. O estudo de plantas medicinais revela espécies de plantas localmente importantes, muitas vezes úteis para a descoberta de novas drogas. O objetivo deste estudo foi conhecer o uso de plantas medicinais em Santa Luzia, Estado do Maranhão, Brasil, e avaliar os padrões sociais e econômicos em relação ao uso da fitoterapia. Os dados etnobotânicos foram coletados por meio de entrevistas semiestruturadas, nas quais participaram 53 moradores (15 homens e 38 mulheres) selecionados aleatoriamente. Um total de 123 espécies de plantas medicinais foram registradas juntamente com seus usos medicinais. Entre as famílias mais citadas, a família Fabaceae apresentou 14 espécies medicinais, seguida por Apiaceae com 7 espécies. As folhas são as partes das plantas usadas com mais frequência e a decocção, o método de preparo. Entre os principais distúrbios tratados com plantas medicinais, as doenças infecciosas e parasitárias e as doenças digestivas foram as mais citadas. Os resultados das correlações entre o número de pessoas por domicílio e a idade dos entrevistados foram positivamente relacionados ao número de plantas citadas. 0 estudo revelou que a área abriga uma diversidade de plantas medicinais e conhecimento associado. As comunidades locais dependem de plantas medicinais para satisfazer suas necessidades básicas de saúde.

\section{Ethnobotanical study of knowledge and medicinal plants use in Santa Luzia, Maranhão, Brazil}

\begin{abstract}
The use of plant species as traditional medicines provides a real substitute in healthcare services for rural communities. The study of medicinal plants reveals locally important plant species often useful for the discovery of new drugs. The aim of this study was to know the use of medicinal plants in Santa Luzia, Maranhão State, Brazil, and to evaluate the social and economic patterns in relation to the use of phytotherapy. Ethnobotanical data were collected using semi-structured interviews in which 53 dwellers ( 15 men and 38 women) randomly selected informants participated. A total of 123 medicinal plant species were recorded together with their medicinal uses. Among the most cited families, the Fabaceae came out as a leading family with 14 medicinal species followed by Apiaceae with 7 species. The leaves are the most commonly used parts of plants and the decoction, the method of preparation. Among the main disorders treated with medicinal plants, infectious and parasitic diseases and digestive diseases were the most cited. The results of the correlations between the number of people per household and the age of respondents are positively related to number of plants cited. The study revealed that the area harbors a diversity of medicinal plants, and associated knowledge. Local communities depend on medicinal plants to meet their basic health care needs.
\end{abstract}

Keywords: Ethnobotany; Medicinal plants; Traditional medicine; Traditional knowledge.

Topic: Uso de Recursos Naturais

Reviewed anonymously in the process of blind peer
Received: $10 / 06 / 2018$

Approved: 24/07/2018
Maria dos Milagres Monsão Gonçalves Instituto Federal do Maranhão, Brasil http://lattes.cnpq.br/6978842219145264 reinaldocajaiba@hotmail.com

Reinaldo Lucas Cajaiba (D)

Instituto Federal do Maranhão, Brasil http://lattes.cnpq.br/4059139430516677 http://orcid.org/0000-0003-0176-9201 reinaldocajaiba@hotmail.com

\section{Wully Barreto dos Santos}

Universidade do Vale do Taquari, Brasil wully bio@hotmail.com
Elson Silva de Sousa (iD

Instituto Federal do Maranhão, Brasil http://lattes.cnpq.br/0079132488060600 http://orcid.org/0000-0002-7369-3704 reinaldocajaiba@hotmail.com

Jesuino da Silva Costa Martins (iD Instituto Federal do Maranhão, Brasil http://lattes.cnpq.br/0872704726864852 http://orcid.org/0000-0003-2137-0054 reinaldocajaiba@hotmail.com

Kleber Sales Pereira

Instituto Federal do Maranhão, Brasil http://lattes.cnpq.br/8768689161696142 reinaldocajaiba@hotmail.com
Vilson de Almeida Sousa

Instituto Federal do Maranhão, Brasil http://lattes.cnpq.br/1956408131769136 http://orcid.org/0000-0002-9636-8357 reinaldocajaiba@hotmail.com MARTINS, J. S. C.; PEREIRA, K. S.; SOUSA, V. A.. Estudo etnobotânico do conhecimento e uso de plantas medicinais em Santa Luzia, Maranhão, Brasil. Revista Ibero Americana de Ciências Ambientais, v.9, n.5, p.12-21, 2018. DOI: http://doi.org/10.6008/CBPC2179$\underline{6858.2018 .005 .0002}$ 


\section{INTRODUÇÃO}

As plantas medicinais têm sido muito utilizadas pelo homem desde os primórdios da humanidade devido suas propriedades medicinais (ARAUJO et al., 2015). A utilização de plantas medicinais como alternativa terapêutica no Brasil é resultante da forte influência cultural indígena, europeia e das tradições africanas (SILVA et al., 2018). O uso de plantas medicinais permanece até os dias atuais, porque os saberes e práticas tradicionais e populares da fitoterapia são repassados de geração a geração; por acreditarem que estas não possuem efeito colateral; difícil acesso aos serviços de saúde, além do baixo custo e facilidade de acesso às plantas medicinais (BEKALO et al., 2009; ARAUJO et al., 2015).

Dados da Organização Mundial de Saúde (OMS) mostram que cerca de $80 \%$ da população mundial faz uso de algum tipo de erva na busca de alívio de alguma sintomatologia ou enfermidade desagradável (LIMA et al., 2011; CAJAIBA et al., 2016a). Segundo Madaleno (2011), no advento do terceiro milênio, cerca de $1 / 3$ da população mundial depende ainda dos poderes curativos das plantas, desse modo, elas são usadas como o único recurso terapêutico de uma parcela da população mundial.

A ciência responsável pelo estudo das plantas e suas relações com a sociedade é a Etnobotânica (GOMES et al., 2014). Segundo Löbler et al. (2014), Etnobotânica é a ciência que estuda as sociedades humanas e suas interações ecológicas, genéticas, evolutivas, simbólicas e culturais. Para Maciel et al. (2008), a Etnobotânica consiste no conhecimento das conceituações desenvolvidas por qualquer sociedade a respeito do mundo vegetal, englobando tanto a maneira como o grupo social classifica as plantas, os usos que são dadas a elas, os místicos, entre outros.

A pesquisa etnobotânica numa comunidade ainda não estudada é uma oportunidade de acessar uma riqueza de informações de cunho etnográfico e futuros recursos biológicos, que poderão servir de subsídios para trabalhos mais focados em determinados aspectos, como, por exemplo, a bioprospecção de novos fármacos ou manejo de áreas de conservação (CAJAIBA et al., 2016b). Sendo o Brasil um país de grande biodiversidade, o mesmo contribui para a adaptação dos vários grupos humanos a riqueza biológica do país, gerando um inestimável sistema de conhecimento local que inclui extensas fontes de informações sobre o uso de plantas utilizadas para fins medicinais (ALVES et al., 2008; SILVA et al., 2018).

Desde o início da colonização do Brasil já se sabe que é frequente o uso de plantas com fins medicinais, entretanto, pouco se sabe sobre o uso de fitoterápicos no estado do Maranhão. Há estudos nessa área realizados para a capital São Luís (GOMES et al., 2014; SANTOS et al., 2017), Bacabal (ARAÚJO et al., 2015), Chapadinha (LIMA, 2016), Presidente Juscelino (MONTELES et al., 2007) e Caxias (OLIVEIRA JÚNIOR et al., 2010; NASCIMENTO et al., 2011). Entretanto, para o município de Santa Luzia, não há estudos nessa área, sendo este o primeiro trabalho realizado, justificando a importância do mesmo. Para preencher essa lacuna, o presente artigo tem por objetivo fazer um levantamento etnobotânico sobre as plantas medicinais utilizadas pelos moradores do povoado Ferro Velho, município de Santa Luzia/MA, a fim de saber quais espécies são utilizadas, os motivos que levam estes moradores a fazerem o uso dessas plantas, as partes das plantas mais utilizadas e as principais doenças tratadas. 


\section{METODOLOGIA}

O estudo foi desenvolvido no povoado de Ferro Velho, município de Santa Luzia, Maranhão. O povoado de Ferro Velho está localizado às margens da Rodovia BR 222, distando aproximadamente $45 \mathrm{Km}$ de Santa Luzia. A principal fonte de renda do povoado é a agricultura de subsistência e bovinocultura.

A coleta dos dados foi realizada durante os meses de setembro a novembro de 2017 através de um questionário semiestruturado composto por 16 abertas e fechadas sobre o uso de plantas medicinais utilizadas pelos entrevistados. As entrevistas foram realizadas nas residências, não havendo critérios para a escolha das pessoas a serem entrevistadas. Foram entrevistadas somente pessoas com idade igual ou superior a 18 anos e, prioritariamente, os responsáveis pelos domicílios pesquisados.

Durante as entrevistas foram recolhidas imagens fotográficas e quando possível, exemplares das espécies citadas para posterior identificação. A identificação das plantas mencionadas foi feita com base na seguinte bibliografia: Lorenzi et al. (2008) e Berg (2010) e pelas bases: International Plant Name Index (IPNI) (http://www.ipni.org), Missouri Botanical Garden (http://www.tropicos.org), Plant Taxonomy Database (http://www.ethnomedicinals.com/searcheng.html) e Lista de Espécies da Flora do Brasil (SOTHERS et al., 2015).

Conforme instruções da Resolução № 466/12 para pesquisas com seres humanos, todas as pessoas entrevistadas durante a execução da pesquisa assinaram um Termo de Consentimento Livre e Esclarecido (TCLE). Para cada espécie citada nas entrevistas foi calculado o Valor de Uso (VU) através da fórmula $V U=\Sigma U i / n$, adaptada por Rossato et al. (1999), sendo $U i=$ soma do número de usos mencionados por cada informante $\mathrm{e}, \mathrm{n}=$ número total de informantes.

Foi calculada a Frequência de indicação do problema pra a espécie (FRIPS), que é resultante da proporção entre a frequência de indicação do problema de saúde para cada espécie (FIPS) pela frequência de citação da espécie correspondente (FCE), através da seguinte fórmula: $F R I P S=(F I P S / F C E) \times 100$. Isto corresponde ao que Amorozo et al. (1988) denominaram 'concordância de uso popular' (CUP). Quanto maior a FRIPS, maior a concordância de uso entre os informantes.

As doenças/sintomas citadas pelos entrevistados foram categorizadas de acordo com a Classificação estatística Internacional de Doenças e problemas relacionados à saúde (CID-10 2013, do inglês International Statistical Classification of Diseases and Related Health Problems - ICD). Para analisar se existe diferença entre o número de plantas citadas entre homens e mulheres foi aplicado o teste não paramétrico do Quiquadrado. Foi aplicada uma correlação de Pearson para verificar a relação entre o número de plantas citadas com escolaridade, renda, idade e número de pessoas por residência.

\section{RESULTADOS}

Foram entrevistadas 53 pessoas com idade variando entre 18 a 86 anos (média de 47,02 \pm 14,44), destes, a maioria eram mulheres (38 entrevistadas). Quanto à escolaridade, a média de estudos foi de quatro anos, sendo que a maioria eram analfabetos $(\approx 51 \%)$. Foi possível verificar in loco que a maior parte dos 
entrevistados são oriundos do próprio estado do Maranhão, principalmente de regiões do interior, onde sua principal fonte de renda é a agricultura de subsistência. Verificou-se ainda que 23 famílias ( $\approx 43 \%)$ tinham mais de cinco filhos.

As plantas medicinais registradas pelos entrevistados estudados foram representadas por 128 espécies distribuídas em 55 famílias botânicas. A maioria das famílias botânicas registradas é representada de uma a quatorze espécies, o que mostra que as plantas medicinais utilizadas não se concentram apenas em poucas famílias e gêneros. As famílias botânicas que apresentaram o maior número de espécies foram Fabaceae (14 espécies), Apiaceae (7 espécies), Lamiaceae e Rutaceae (6 espécies cada). As espécies de plantas medicinais que obtiveram o maior Valor de Uso - VU foram: Malva-do-reino (Malva sylvestris, VU = 0,63 ), Hortelã (Mentha crispa, $\mathrm{VU}=0,54$ ), Vique (Mentha arvensis, $\mathrm{VU}=0,32$ ) e Gengibre (Zingiber offcinale, $\mathrm{VU}=0,28)$.

No que se refere à frequência relativa de indicação para a espécie (FRIPS), 15 espécies de plantas apresentaram valores acima de $50 \%$, representando aproximadamente $29 \%$ do total, das quais destacam-se M. sylvestris (Malva do reino, FRIPS $=97 \%)$; . crispa $($ Hortelã, FRIPS $=88 \%) ;$ L. alba $($ Erva-cidreira, FRIPS $=$ 81\%) (Tabela 1).

Tabela 1: Usos principais, nome popular/científico e Frequência relativa de indicação para cada espécie (FRIPS).

\begin{tabular}{|l|l|l|l|}
\hline Especie & Nome popular & Indicação & FRIPS* \\
\hline M. sylvestris & Malva do reino & Gripe; resfriado & $97 \%$ \\
\hline M. crispa & Hortelã & Gripe; resfriado & $88 \%$ \\
\hline L. alba & Erva-cidreira & Calmante & $81 \%$ \\
\hline M. arvensis & Vique & Gripe; tosse & $66 \%$ \\
\hline A. vera & Babosa & Inflamação; cicatrizante & $59 \%$ \\
\hline C. citratus & Capim-santo & Calmante & $58 \%$ \\
\hline Z. offcinale & Gengibre & Inflamação na garganta; gripe & $54 \%$ \\
\hline P. granatum & Romã & Hemorragia; infecção & $53 \%$ \\
\hline K. pinnata & Folha santa & Anti-inflamatório & $53 \%$ \\
\hline M. chamomilla & Camomila & Calmante & $52 \%$ \\
\hline M. arvensis & Vique & Gripe; & $51 \%$ \\
\hline C. ambrosioides & Mastruz & Anti-inflamatório; cicatrizante & $51 \%$ \\
\hline V. condensata & Boldo & Dor estomacal; fígado & $50 \%$ \\
\hline E. melanophloia & Eucalipto & Gripe; resfriado & $50 \%$ \\
\hline L. alba & Erva-cidreira & Calmante & $50 \%$ \\
\hline
\end{tabular}

*Espécies de plantas que apresentaram FRIPS acima de 50\%.

Quanto a parte vegetal utilizada nas preparações dos remédios caseiros, observou-se uma maior utilização das folhas ( $35 \%$ das citações), seguida por entrecasca ( $20 \%$ das citações) e raiz ( $13 \%$ das citações). Com relação ao modo de preparo, a técnica mais utilizada entre os entrevistados, foi em forma de chá (59\%), e a forma de uso mais frequente das plantas medicinais é através da ingestão (87\%), seja através da alimentação, chás, suco, xarope ou garrafadas.

As indicações terapêuticas que são tratadas através das 123 espécies de plantas medicinais citadas pelos entrevistados foram agrupadas em 13 categorias de sintomas/doenças de acordo com a CID-10, sendo que as doenças infecciosas e parasitárias - DIP foram as mais prevalentes $(27 \%)$ seguida por doenças do aparelho digestivo - DAD (16\%) e doenças do aparelho respiratório - DAR (13\%) (Figura 1). 


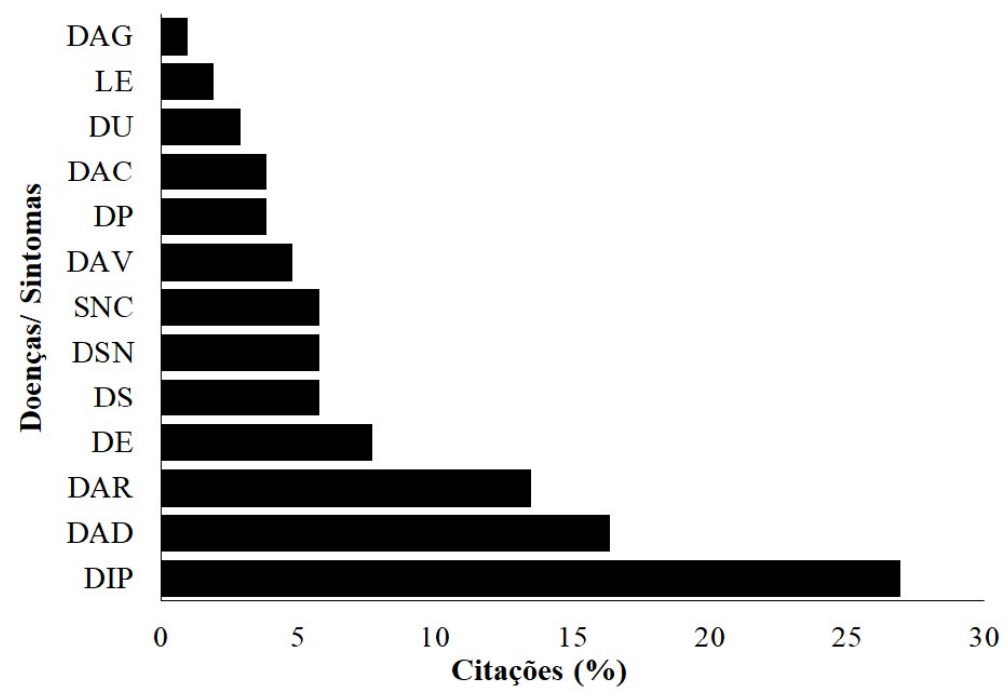

Figura 1: Porcentagem de citações das plantas medicinais indicadas pelos informantes por categorias de doenças segundo o CID 10, no povoado Ferro Velho, Santa Luzia, Maranhão. DAG= Doenças do aparelho geniturinário; LE= Lesões e envenenamento; $\mathrm{DU}=$ Doenças urinárias; $\mathrm{DAC}=$ Doenças do aparelho circulatório; $\mathrm{DP}=\mathrm{Doenças} \mathrm{da} \mathrm{pele;} \mathrm{DAV}=$ Doenças do aparelho vascular; SNC= Sintomas não classificados; DSN= Doenças do sistema nervoso; DS=Doenças do sangue; $\mathrm{DE}=$ Doenças endócrinas; $\mathrm{DAR}=$ Doenças do aparelho respiratório; $\mathrm{DAD}=$ Doenças do aparelho digestivo; $\mathrm{DIP}=$ Doenças infecciosas e parasitárias.

Com relação a forma de obtenção de conhecimentos sobre o uso de plantas medicinais, $42 \%$ dos entrevistados citaram os familiares, seguidos por amigos (29\%), livros (14\%) e médicos (11\%). O tempo de uso das plantas medicinais pelos entrevistados teve uma média de 21,13 anos, sendo utilizada cerca de 19,11 vezes ao ano. O principal fator que leva os entrevistados a fazerem o uso de plantas medicinais é devido esse tipo de medicamento ser de fácil acesso/obtenção com aproximadamente 34\% das citações, seguido por baixo custo (23\%), ausência de efeitos colaterais (16\%) e serem considerados naturais (11\%).

O teste do Qui-quadrado mostrou que as mulheres conhecem um maior número de plantas para o tratamento de doenças do que os homens $\left(\chi^{2} 54,28, p<0,01\right)$. A correlação de Pearson mostrou que houve correlação positiva entre o número de plantas citadas com a idade $\left(r_{s}=0,53, p<0,05\right)$, baixa escolaridade $\left(r_{s}\right.$ $=0,48, p<0,05)$ e o número de filhos por residência $\left(r_{s}=0,62, p<0,05\right)$.

\section{DISCUSSÃO}

Um número considerável (128 espécies) de plantas medicinais foi documentado neste estudo. Nossos resultados demonstram que o número de plantas medicinais utilizadas pela população estudada é uma alternativa importante no tratamento de doenças, sendo utilizada principalmente por serem um medicamento de fácil acesso, baixo custo e serem considerados como "naturais". Assim, é a primeira opção de tratamento de saúde para a população estudada que sofre principalmente por falta de acesso aos sistemas de saúde pública. Dessa forma, o presente estudo representa uma documentação útil, que pode contribuir para a preservação do conhecimento sobre o uso de plantas medicinais nesta região.

Entre as famílias botânicas, aquela que apresentou o maior número de espécies foi Fabaceae, com 14 espécies, seguida por Apiaceae, com 7 espécies. $O$ achado da família Fabaceae como contribuinte de maior número de espécies de plantas usadas para fins medicinais do que outras famílias estão de acordo com outros estudos (YINEGER et al., 2007; HAILEMARIAM et al., 2009; MEGERSA et al., 2013). Este fato, por ser 
justificado, por que essas famílias são culturalmente importantes, e estão amplamente distribuídas em regiões temperadas e tropicais do mundo (BENNETT et al., 2000; CAJAIBA et al., 2016a). Há evidências de que a seleção de plantas para uso medicinal não é feita ao acaso e que famílias botânicas com compostos bioativos tendem a ser mais bem representadas nas farmacopeias populares (MOERMAN et al., 2003).

Com relação às espécies mais citadas, a $M$. sylvestris e $M$. crispa apresentaram maior VU e FRIPS. Essas plantas também foram as mais citadas em outros estudos (SILVA et al., 2018), sendo utilizadas principalmente para o tratamento de gripe/resfriado/garganta, confirmando o reconhecimento desses recursos vegetais para o tratamento dessas doenças nas comunidades estudadas. $O$ fato de algumas das plantas relatadas terem usos semelhantes em outros lugares pode ser considerado como indicação de sua eficácia farmacológica (HAILEMARIAM et al., 2009; MEGERSA et al., 2013). Ressalta-se que os altos índices de VU e FRIPS indicam que as propriedades medicinais das espécies citadas para estas categorias merecem estudos mais aprofundados (ALVES et al., 2013; AZEVEDO et al., 2007) e dão boas indicações sobre espécies que podem servir para problemas particulares de saúde. Essas metodologias são importantes indicadores da possível atividade farmacológica das espécies, pois quanto mais informantes indicarem determinado uso, mais segurança haverá na validação destas informações, podendo no futuro, servir como referência para estudos farmacológicos na busca de novas drogas (ALVES et al., 2013; HAILEMARIAM et al., 2009; ZENEBE et al., 2012).

Quanto a parte vegetal utilizada nas preparações dos remédios caseiros, observou-se uma maior utilização das folhas (35\% das citações). Estes resultados estão de acordo com vários estudos realizados sobre etnobotânica, onde as folhas são as partes mais utilizadas (ADEFA, 2012; ZENEBE et al., 2012; MEKUANENT et al., 2015). Isso se deve ao fato de as folhas serem coletadas com mais facilidade além de serem encontradas em praticamente o ano todo, já que flores, frutos e sementes não se encontram disponíveis em todas as épocas do ano (SILVA et al., 2018). Além disso, a utilização de folhas pode não causar um efeito prejudicial às plantas em comparação com o uso de raízes, caules e materiais reprodutivos (flores, frutos e sementes) (HONG et al., 2015). Estudos mostraram que a remoção de até $50 \%$ das folhas das árvores não afeta significativamente o crescimento das plantas (POFFENBERGER et al., 1992), no entanto, havia plantas medicinais na área de estudo em que as raízes, rizomas, cascas e caules eram coletadas para o tratamento de doenças. Como resultado, a coleta excessiva representa uma ameaça do ponto de vista ecológico e da sobrevivência das plantas-mãe (HUNDE et al., 2006; ZHENG et al., 2009; ZENEBE et al., 2012; MEGERSA et al., 2013; UMAIR et al., 2017).

Com relação ao modo de preparo, a técnica mais utilizada entre os entrevistados foi em forma de chá, seja por infusão ou decocção e a forma de uso mais frequente das plantas medicinais é através da ingestão, seja através da alimentação, chás, suco, xarope ou garrafadas, corroborando com vários estudos (LEÃO et al., 2007; OLIVEIRA et al., 2012; SANTOS et al., 2016). A decocção é usada como uma das principais práticas para preparar o medicamento no sistema de cura tradicional, porque é fácil de fazer misturando-se com chá, água ou sopa (SSEGAWA et al., 2007; ROKAYA et al., 2010). Ao fazer a decocção, o material vegetal é fervido em água até o volume de água ser reduzida a um quarto do seu volume original (KAYANI et al., 
2014), enquanto o extrato bruto é obtido espremendo ou esmagando as partes da planta (CECHINEL-FILHO, 2012). De acordo com Castellani (1999), a infusão é utilizada em todas as partes de plantas medicinais macias, tais como as flores, folhas e botões, uma vez que são ricas em componentes voláteis, aromas suaves e princípios ativos que se degradam pela ação combinada da água e do calor prolongado.

As indicações terapêuticas que são tratadas através das 128 espécies de plantas medicinais citadas pelos entrevistos foram agrupadas em 13 categorias de sintomas/doenças de acordo com a CID-10, sendo que as doenças infecciosas e parasitárias - DIP, doenças do aparelho digestivo - DAD e doenças do aparelho respiratório - DAR foram as mais prevalentes. Esses resultados são concordantes com os de Baptistel et al. (2014) e Silva et al. (2018) que também encontraram um maior número de indicações para problemas do sistema digestório e doenças infecciosas e parasitárias. Comumente, os sintomas referentes a estas classes estão relacionados com os problemas primários de saúde, que num primeiro momento são tratados com o uso de plantas medicinais e sem auxílio do médico (POSSAMAI, 2000). Esses resultados podem relacionar-se à falta de saneamento básico na região, como tratamento de água, esgotos e coletas dos resíduos, uma vez que o povoado não apresenta essas infraestruturas. Essas tipologias de doenças são comuns, principalmente nas regiões do interior do país, assim como em outros países da América Latina (BENNETT \& PRANCE, 2000).

Nossos resultados demonstraram que a principal forma de obtenção de conhecimento sobre o uso de plantas medicinais, são os familiares e amigos (juntos somam aproximadamente $71 \%$ das citações). Verificamos também que as mulheres conhecem mais espécies de plantas e formas de tratamento e que as pessoas mais idosas, famílias de baixa renda e com maior número de filhos tendem a conhecer ou utilizar uma maior diversidade plantas medicinais. Estes resultados reforçam o arcabouço cultural para o uso das plantas medicinais e sua transmissão de geração para geração, além de corroborar com estudos realizados tanto no Brasil como em outras partes do mundo (TEKLEHAYMANOT, 2009; BENITEZ et al., 2010; ADEFA, 2012; ZENEBE et al., 2012; AKDIME et al., 2015; CAJAIBA et al., 2016b).

Pesquisas têm demonstrado que o conhecimento do uso de plantas medicinais está quase desaparecendo entre a geração jovem (TEKLEHAYMANOT, 2009) e isso pode ser justificado por vários motivos: 1) as pessoas mais idosas não tenham repassado adequadamente o conhecimento para a próxima geração. Assim, a geração jovem pode não ter a oportunidade de adquirir o conhecimento tradicional (TEKLEHAYMANOT, 2009); 2) cautela dos idosos em manter seus conhecimentos em segredo (ABEBE et al., 1993), podendo este valioso conhecimento ser rapidamente desgastado quando esses idosos morrem (MEKUANENT et al., 2015); 3) devido ao interesse desvanecido dos jovens (ZENEBE et al., 2012), sendo afetados principalmente pela modernização (acesso à educação moderna e serviços de saúde) e mudanças ambientais (HUNDE et al., 2004; BEKALO et al., 2009) e até mesmo por descrenças (SILVA et al., 2018). Dessa forma, tal estudo se faz importante por disseminar para a sociedade a importância das plantas medicinais.

\section{CONCLUSÕES}

Os resultados deste estudo revelaram que há uma alta diversidade de plantas medicinais e conhecimentos tradicionais sobre o uso e preparação que ainda é mantido entre as pessoas da região 
estudada. No entanto, é preocupante a constatação de que o conhecimento sobre remédios fitoterápicos ser realizado principalmente por pessoas mais idosas, o que pode levar a um declínio no uso de plantas medicinais por gerações mais jovens e levar ao desaparecimento do conhecimento tradicional associado às plantas. Pesquisas para essa região ainda são escassas e se fazem necessários mais estudos com distintas abordagens para somar aos resultados aqui apresentados. Para estudos futuros, sugere-se, portanto, que pesquisas sejam realizadas com diferentes populações do município, tais como ribeirinhos, moradores de assentamentos rurais, distritos do município e populações indígenas.

Nossos resultados apresentam um valor significativo para gerentes de conservação e gestores públicos para o manejo sustentável de espécies de plantas medicinais, visto que a exploração excessiva poderá trazer prejuízos para a biodiversidade. Dessa forma, é imperativo a sensibilização dos usuários e comunidade em geral por meio de treinamento ou educação sobre a utilização sustentável e o gerenciamento dos recursos vegetais em geral e das plantas medicinais em particular.

Por fim, as diferentes espécies vegetais observadas neste trabalho indicam a preferência da comunidade local estudada na utilização de plantas medicinais no tratamento de doenças específicas, bem como apresenta o conhecimento da medicina tradicional dessa população. Portanto, as plantas medicinais aqui documentadas podem servir de base para pesquisas futuras para a identificação de bioativos, atividades biológicas in vivo/in vitro, o que pode levar a novos e potenciais medicamentos.

\section{REFERÊNCIAS}

ABEBE, D.; AYEHU, A.. Medicinal plants and enigmatic health practices of northern Ethiopia. Addis Ababa: B.S.P.E, 1993.

ADEFA, M.. Therapeutic uses of Traditional Medicinal Plants in North Ethiopia: A case study of Tehuledere District, South Wollo, Ethiopia. International Journal of Biomedical Research and Analysis, v.3, n.1, p.15-22, 2012.

AKDIME, H.; BOUKHIRA, S.; EL MANSOURI, L.; HAMSAS EL YOUBI, A.; BOUSTA, D.. Ethnobotanical study and traditional knowledge of medicinal plants in Ain Leuh Region (MiddleAtlas of Morocco). American Journal of Advanced Drug Delivery, v.3, n.3, p.248-263, 2015.

ALVES, G. S. P.; POVH, J. A.. Estudo etnobotânico de plantas medicinais na comunidade de Santa Rita, Ituiutaba/MG. Biotemas, v.26, n.3, p.231-42, 2013.

ALVES, R. R. N.; SILVA, C. C.; ALVES, H. N.. Aspectos socioeconômicos do comercio de plantas e animais medicinais em áreas metropolitanas do Norte e Nordeste do Brasil. Revista de Biologia e Ciências da Terra, v.8, n.1, p.181-189, 2008.

AMOROZO, M. C. M.; GÉLY, A.. Uso de plantas medicinais por caboclos do Baixo Amazonas, Barcarena, Pará, Brasil. Brasil. Boletim do Museu Paraense Emílio Goeldi, v.4, n.1, p.47-131, 1988.

ARAÚJO, I. F. M.; SOUZA, L. F.; GUARÇONI, E. A. E.; FIRMO, W. C. A.. O comércio de plantas com propriedades medicinais na cidade de Bacabal, Maranhão, Brasil. Natureza Online, v.13, n.3, p.111-116, 2015.

ARAÚJO, J. L.; LEMOS, J. R.. Estudo etnobotânico sobre plantas medicinais na comunidade de Curral Velho, Luís Correia, Piauí, Brasil. Biotemas, v.28, n.2, p.125-136, 2015.

AZEVEDO, V.; KRUEL, V. S.. Plantas medicinais e ritualísticas vendidas em feiras livres no Município do Rio de Janeiro, RJ Brasil: estudo de caso nas zonas Norte e Sul. Acta Botanica Brasilica, v.21, n.2, p.263-75, 2007.

BAPTISTEL, A. C.; COUTINHO, J. M. C. P.; LINS NETO, E. M. F.; MONTEIRO, J.M.. Plantas medicinais utilizadas na Comunidade Santo Antônio, Currais, Sul do Piauí: um enfoque etnobotânico. Revista Brasileira de Plantas Medicinais, v.16, n.2, p.406-25, 2014.

BEKALO, T. H.; WOODMATAS, S. D.; WOLDEMARIAM, Z. A.. An ethnobotanical study of medicinal plants used by local people in the lowlands of Konta Special Woreda, southern nations, nationalities and peoples regional state, Ethiopia. Journal of Ethnobiology and Ethnomedicine, v.5, n.26, 1-15, 2009.

BENITEZ, G.; GONZALEZ-TEJERO, M. R.; MOLERO-MESA, J.. Pharmaceutical ethnobotany in the western part of Granada province (southern Spain): Ethnopharmacological synthesis. Journal of Ethnopharmacology, v.129, n.1, p.87-105, 2010.

BENNETT, B. C.; PRANCE, G. T.. Introduced plants in the indigenous pharmacopoeia of Northern South America. Economic Botany, v.54, n.1., p.90-102, 2000. 
BERG, M. E.. Plantas medicinais na Amazônia: contribuição ao seu conhecimento sistemático. 3a Edição. Belém-PA: Museu Emílio Goeldi, 2010. 269 p.

CAJAIBA, R. L.; GOMES, A. F.; SANTOS, M. C.; MEDEIROS, R. R.; SILVA, W. B.. Perfil dos comerciantes de plantas medicinais no município de Uruará, Pará, Brasil. Enciclopédia Biosfera, v.13, n.24, p.1473-1482, 2016b.

CAJAIBA, R. L.; SILVA, W. B.; SOUSA, R. D. N.; SOUSA, A.S .. Levantamento etnobotânico de plantas medicinais comercializadas no município de Uruará, Pará, Brasil. Biotemas, v.29, n.1, p.115-131, 2016a.

CASTELLANI, D. C.. Plantas medicinais. Agromídia software: Viçosa. 1999. 20 p.

CECHINEL-FILHO, V.. Plant bioactives and drug discovery: principles, practice, and perspectives: John Wiley \& Sons 2012.

GOMES, L. G.. 'Comércio étnico' em Belleville: memória, hospitalidade e conveniência. Revista Estudos Históricos, v.1, n.29, p. 187-207, 2002.

GOMES, P. R. M.; FIRMO, W. C. A.; VILANOVA, C. M.. Estudo etnobotânico de plantas medicinais hipoglicemiantes no bairro Maracanã no município de São Luís, Maranhão, Brasil. Scientia Plena, n.10, n.9, p.1-11, 2014.

\section{HAILEMARIAM, T.; DEMISSEW, S.; ASFAW, Z.. An} ethnobotanical study of medicinal plants used by local people in the lowlands of Konta special Wereda, Southern Nations, Nationalities and People Regional State, Ethiopia. Journal of Ethnobiology and Ethnomedicine, v.6, n.25, p.115, 2009.

HONG, L.; GUO, Z.; HUANG, K.; WEI, S.; LIU, B.; MENG, S.; LONG, C.. Ethnobotanical study on medicinal plants used by Maonan people in China. Journal of Ethnobiology and Ethnomedicine, v.11, n.32, p.1-35, 2015.

HUNDE, D.; ASFAW, Z.; KELBESSA, E.. Use and Management of Ethnoveterinary Medicinal Plants by Indigenous People in 'Boosat', Wolenchiti Area. Ethiopian Journal of Biological Science, v.3, n.2, p.113-132, 2004.

KAYANI, S.; AHMAD, M.; ZAFAR, M.; SULTANA, S.; KHAN, M. P. Z.; ASHRAF, M. A.; HUSSAIN, J.; YASEEN, G..

Ethnobotanical uses of medicinal plants for respiratory disorders among the inhabitants of Gallies-Abbottabad, Northern Pakistan. Journal of Ethnopharmacology, v.156, n.1, p.47-60, 2014.

LEÃO, R. B. A.; FERREIRA, M. R. C.; JARDIM, M. A. G.. Levantamento de plantas de uso terapêutico no município de Santa Bárbara do Pará, Estado do Pará, Brasil. Revista Brasileira de Farmácia, v.88, n.1, p.21-25, 2007.

LIMA, E.. Levantamento etnobotânico em áreas de Cerrado: Resgatando conhecimentos populares acerca do uso das plantas medicinais no município de Chapadinha, MA, Brasil. Monografia (Graduação) - Universidade Federal do Maranhão, 2016.
LIMA, R. A.; MAGALHÃES, A. S.; SANTOS, M. R. A.

Levantamento etnobotânico de plantas medicinais utilizadas na cidade de Vilhena, Rondônia. Revista Pesquisa \& Criação, v.10, n.2, p.165-179, 2011.

LÖBLER, L.; SANTOS, D.; RODRIGUES, E. S.; SANTOS, N. R. Z.. Levantamento etnobotânico de plantas medicinais no bairro Três de Outubro da cidade de São Gabriel, RS, Brasil. Revista Brasileira de Biociências, v.12, n.2, p.81-89, 2014.

LORENZI, H.; MATOS, F. J. A.. Plantas medicinais no Brasil: nativas e exóticas cultivadas. 2 ed. Nova Odessa: Instituto Plantarum, 2008.

MACIEL, M.A.M.; PINTO, A.C.; VEIGA JR, V.F.. Plantas medicinais: A necessidade de estudos multidisciplinares. Química Nova, v.25, n.3, p.429-438, 2002.

MADALENO, I. M.. Plantas da medicina popular de São Luís, Brasil. Boletim do Museu Paraense Emílio Goeldi, v.6, n.2, p.273-286, 2011.

MEGERSA, M.; ASFAW, Z.; KELBESSA, E.; BEYENE, A.; WOLDEAB, B.. An ethnobotanical study of medicinal plants in Wayu Tuka District, East Welega Zone of Oromia Regional State, West Ethiopia. Journal of Ethnobiology and Ethnomedicine, v.9, n.1, 1-18, 2013.

MEKUANENT, T.; ZEBENE, A.; SOLOMON, Z.. Ethnobotanical Study of Medicinal Plants in Chilga District, Northwestern Ethiopia. Journal of Natural Remedies, v.15, n.2, p.88-112, 2015.

MOERMAN, D. E.; ESTABROOK, G. F.. Native Americans' choice of species for medicinal use is dependent on plant family: conirmation with meta-signiicance analysis. Journal of Ethnopharmacology, v.87, n.1, p.51-59, 2003.

MONTELES, R.; PINHEIRO, C. U. B.. Plantas medicinais em um quilombo maranhense: uma perspectiva etnobotânica. Revista de Biologia e Ciências da Terra, v.7, n.2, p.38-48, 2007.

NASCIMENTO, J. M.; CONCEIÇÃO, G. M.. Plantas medicinais e indicações terapêuticas da comunidade quilombola Olho d'água do Raposo, Caxias, Maranhão, Brasil. Revista de Biologia e Farmácia, v.6, n.2, p.138- 151, 2011.

OLIVEIRA JÚNIOR, S. R.; CONCEIÇÃO, G. M.. Espécies vegetais nativas do cerrado utilizadas como medicinais pela Comunidade Brejinho, Caxias, Maranhão, Brasil. Cadernos de Geociências, v.7, n.2, 2010.

OLIVEIRA, E. R.; MENINI NETO, L.. Levantamento etnobotânico de plantas medicinais utilizadas pelos moradores do povoado de Manejo, Lima Duarte/MG. Revista Brasileira de Plantas Medicinais, v.14, n.1., p.311 20, 2012.

POFFENBERGER, M.; MCGEAN, B.; RAVINDRANATH, N. H.; GADGIL, M.. Editors of Field Methods Manual. Volume 1: Diagnostic tools for supporting joint forest management systems. Society for Promotion of Wastelands Development, New Delhi, India, 1992.

POSSAMAI, R. M.. Levantamento etnobotânico das plantas de uso medicinal em Mariana Pimentel, RS. [Dissertação]. 
Porto Alegre: Universidade Federal do Rio Grande do Sul. 2000.

ROKAYA, M.B.; MÜNZBERGOVÁ, Z.; TIMSINA, B. Ethnobotanical study of medicinal plants from the Humla district of western Nepal. Journal of Ethnopharmacology, v.130, n.3, p.485-504, 2010.

ROSSATO, S. C.; LEITAO FILHO, H.; BEGOSSI, A.. Ethnobotany of caiçaras of the Atlantic Forest Coast (Brazil). Economic Botany, v.53, n.4, p.387-395, 1999.

SANTOS, J. X.; REIS, A. R. S.; MATOS, A. S.; LEÃO, F. M.; CARVALHO, J. C.. Caracterização etnobotânica de essências florestais com fins medicinais utilizadas pela Etnia Xipaya, no município de Altamira/PA. Biota Amazônia, v.6, n.2, p.1-8, 2016.

SANTOS, K. A.; VILANOVA, C. M.. Estudo etnobotânico de plantas medicinais utilizadas como hipoglicemiantes por usuários do Programa de Fitoterapia da Universidade Federal do Maranhão, Brasil. Scientia Plena, v.13, n.3, p.112, 2017.

SILVA, W. B.; CAJAIBA, R. L.; PARRY, M. M.. Levantamento etnobotânico de plantas medicinais utilizadas pelos moradores do município de Uruará, estado do Pará, Brasil. Revista Cubana de Plantas Medicinales, v.29, n.1, p.115-
131, 2018. DOI: https://doi.org/10.5007/2175$\underline{7925.2016 \mathrm{~V} 29 \mathrm{n} 1 \mathrm{p} 115}$

SSEGAWA, P.; KASENENE, J. M.. Medicinal plant diversity and uses in the Sango bay area, Southern Uganda. Journal of Ethnopharmacology, v.113, n.3, p.521-540, 2007.

TEKLEHAYMANOT, T.. Ethnobotanical study of knowledge and medicinal plants use by the people in Dek Island in Ethiopia. Journal of Ethnopharmacology, v.124, n.1, p.6978, 2009.

UMAIR, M.; ALTAF, M.; ABBASI, A. M.. An ethnobotanical survey of indigenous medicinal plants in Hafizabad district, Punjab-Pakistan. PLoS ONE, v.12, n.6, 2017.

YINEGER, H.; YEHUWALAW, D.. Traditional medicinal plant knowledge and use by local healers in Sekoru District, Jimma Zone, Southwestern Ethiopia. Journal of Ethnobiology and Ethnomedicine, v.3, n.24, p.1-7, 2007.

ZENEBE, G.; ZERIHUN, M.; SOLOMON, Z.. An Ethnobotanical Study of Medicinal Plants in Asgede Tsimbila District, Northwestern Tigray, Northern Ethiopia. Ethnobotany Research \& Applications, v.10, n.1, p.305-320, 2012.

ZHENG, X.; XING, F.. Ethnobotanical study on medicinal plants around Mt. Yinggeling, Hainan Island, China. Journal of Ethnopharmacology, v.124, n.2, p.197-210, 2009.

A CBPC - Companhia Brasileira de Produção Científica (CNPJ: 11.221.422/0001-03) detém os direitos materiais desta publicação. Os direitos referem-se à publicação do trabalho em qualquer parte do mundo, incluindo os direitos às renovações, expansões e disseminações da contribuição, bem como outros direitos subsidiários. Todos os trabalhos publicados eletronicamente poderão posteriormente ser publicados em coletâneas impressas sob coordenação da Sustenere Publishing, da Companhia Brasileira de Produção Científica e seus parceiros autorizados. Os (as) autores (as) preservam os direitos autorais, mas não têm permissão para a publicação da contribuição em outro meio, impresso ou digital, em português ou em tradução. 